\title{
Pacemaker lead-associated tricuspid regurgitation in patients with or without pre-existing right ventricular dilatation
}

\author{
Martin Riesenhuber ${ }^{1}$. Andreas Spannbauer ${ }^{1}$. Marianne Gwechenberger ${ }^{1}$. Thomas Pezawas ${ }^{1}$. Christoph Schukro ${ }^{1}$. \\ Günter Stix ${ }^{1}$. Matthias Schneider ${ }^{1}$. Georg Goliasch ${ }^{1}$ - Anahit Anvari ${ }^{1} \cdot$ Thomas Wrba $^{2}$. Cesar Khazen ${ }^{3}$. \\ Martin Andreas ${ }^{3}$. Günther Laufer ${ }^{3}$. Christian Hengstenberg ${ }^{1} \cdot$ Mariann Gyongyosi $^{1,4}$
}

Received: 12 November 2020 / Accepted: 28 January 2021 / Published online: 10 February 2021

(c) The Author(s) 2021

\begin{abstract}
Background Transcatheter tricuspid valve intervention became an option for pacemaker lead-associated tricuspid regurgitation. This study investigated the progression of tricuspid regurgitation (TR) in patients with or without pre-existing right ventricular dilatation (RVD) undergoing pacemaker implantation.

Methods Patients were included if they had implantation of transtricuspid pacemaker lead and completed echocardiography before and after implantation. The cohort was divided in patients with and without RVD (cut-off basal RV diameter $\geq 42 \mathrm{~mm}$ ). TR was graded in none/mild, moderate, and severe. Worsening of one grade was defined as progression. Survival analyses were plotted for 10 years.

Results In total, 990 patients were analyzed (24.5\% with RVD). Progression of TR occurred in $46.1 \%$ of patients with RVD and in $25.6 \%$ of patients without RVD $(P<0.001)$. Predictors for TR progression were RV dilatation (OR 2.04; 95\% CI 1.27-3.29; $P=0.003$ ), pre-existing TR (OR 4.30; 95\% CI 2.51-7.38; $P<0.001$ ), female sex (OR 1.68; 95\% CI 1.16-2.43; $P=0.006$ ), single RV lead (OR 1.67; 95\% CI 1.09-2.56; $P=0.018$ ), mitral regurgitation (OR 2.08; 95\% CI 1.42-3.05; $P<0.001$ ), and enlarged left atrium (OR 1.98; 95\% CI 1.07-3.67; $P=0.03$ ). Survival-predictors were pacemaker leadassociated TR (HR 1.38; 95\% CI 1.04-1.84; $P=0.028$ ), mitral regurgitation (HR 1.34; 95\% CI 1.02-1.77; $P=0.034$ ), heart failure (HR 1.75; 95\% CI 1.31-2.33; $P<0.001$ ), kidney disease (HR 1.62; 95\% CI 1.25-2.11; $P<0.001$ ), and age $\geq 80$ years (HR 2.84; 95\% CI 2.17-3.71; $P<0.001$ ).
\end{abstract}

Conclusions Patients with RVD receiving pacemaker suffered from increased TR progression, leading to decreased survival.

Mariann Gyongyosi

mariann.gyongyosi@meduniwien.ac.at

1 Department of Cardiology, Medical University of Vienna,

Vienna, Austria

2 Medical University of Vienna, IT Systems

and Communications, Vienna, Austria

3 Department of Cardiac Surgery, Medical University of Vienna, Vienna, Austria

4 Department of Internal Medicine II, Division of Cardiology, Medical University of Vienna, Währinger Gürtel 18-20, 1090 Vienna, Austria 


\section{Graphic abstract}

Pacemaker Lead-associated Tricuspid Regurgitation in Patients With or Without Pre-existing Right Ventricular Dilatation

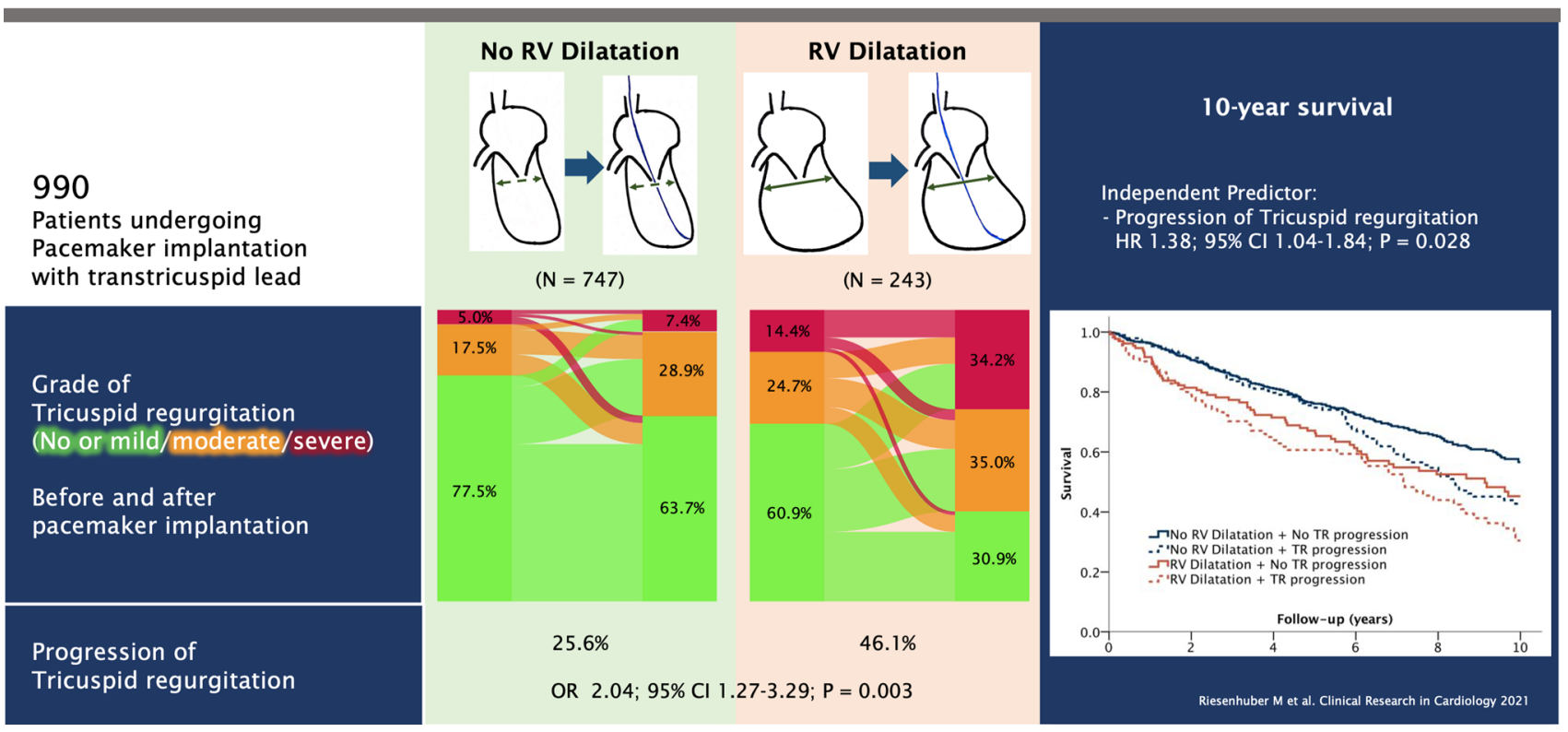

Keywords Tricuspid regurgitation $\cdot$ Pacemaker $\cdot$ Right ventricle $\cdot$ Valvular heart disease $\cdot$ Device complications

\section{Introduction}

Tricuspid regurgitation (TR) represents a major burden of cardiovascular disease, and the prevalence of TR is comparable to the prevalence of aortic stenosis [1]. Approximately three million patients in Europe and 1.6 million patients in the United States suffer from clinically relevant TR [2]. TR is an independent risk factor for increased mortality [3], and surgical treatment of TR is associated with significant perioperative mortality $[4,5]$. Therefore, concepts for efficient percutaneous interventional treatments of TR are on the rise. Transcatheter tricuspid valve interventions (TTVI) represent a safe, effective, and alternative treatment approach for secondary TR [6,7], and improve the severity of secondary TR, symptoms, and hospitalizations for heart failure [8-10]. However, TTVI is offered only in tertiary centers and is not yet standard of care.

Interference of a right ventricular transtricuspid pacing lead with the tricuspid valve might contribute to or cause $\mathrm{TR}$, and is reported in $7 \%$ to $45 \%$ of RV lead implantations [11]. RV leads of cardiac implantable electronic devices (CIEDs) are associated with progression of TR, which is associated with poor outcomes [12-17]. Lead-associated TR affects long-term RV function, which is linked to decreased survival [18].

CIED-induced TR can be divided in primary and secondary CIED-induced TR, and recent data found up to
$60 \%$ of worsened TR after CIED implantation are of secondary origin [19]. While primary CIED-induced TR is caused by direct interaction of the lead and the tricuspid valve, secondary CIED-induced TR has its origin in RV dilatation due to pacing/heart failure. Untreated primary CIED-induced TR triggers RV dilatation due to volume overload, and leads to secondary TR. If this "point of no return" is reached, lead extraction could not reverse TR $[19,20]$.

Currently, only one representative study is published addressing TTVI in patients with CIEDs: Taramasso et al. analyzed 470 patients with severe TR undergoing TTVI, and compared patients with and without CIED [21]. Equal treatment outcomes could be demonstrated in terms of rates of procedural success, residual TR, symptomatic improvement, and survival.

In the era of increasing TTVI, CIED-induced TR has to be critically re-evaluated with special attention for primary and secondary CIED-induced TR. Considering predictable CIED-induced TR, leadless pacing could become of interest. If secondary CIED-induced TR occurs, TTVI could be an effective treatment strategy.

The aim of this study was to evaluate pacemaker leadassociated TR regarding right heart morphology (dilated vs. non-dilated RV) and identify predicting factors for pacemaker lead-associated TR and survival. 


\section{Methods}

\section{Study design}

This retrospective cohort study is based on patients of the Department of Cardiology at the Medical University of Vienna, who had a first pacemaker implantation and echocardiographic studies 17 months (IQR 2-55) before and 5 months (IQR 0.1-21) after the implantation.

This study followed all principles of the Declaration of Helsinki and was approved by the Ethics Committee of the Medical University of Vienna (EK 1525/2015).

\section{Patients}

All patients with implantation of a cardiac pacemaker with a transtricuspid pacing lead were included in the study, if they had an echocardiography before and after pacemaker implantation. Patients were enrolled from May 2000 to April 2015. Patients with implantable cardioverter defibrillator (ICD), cardiac resynchronization therapy (CRT) or singlechamber pacemakers with only an atrial pacing lead were excluded from the study.

\section{Baseline parameters}

The comorbidities (coronary artery disease, heart failure, diabetes mellitus, myocarditis, "any" atherosclerosis, previous stroke or transient ischemic attack (TIA), previous coronary artery bypass grafting $(\mathrm{CABG})$, atrial fibrillation, chronic kidney disease, endocarditis) were derived from the hospital information system based on ICD-10 codes. Patients with heart failure included those with reduced or preserved ejection fraction. The diagnosis of diabetes included patients with type 1 or type 2 diabetes.

\section{Clinical follow-up of the patients}

Clinical follow-up up to 10 years was available in 562 patients, and 5569 person-years were analyzed. Mortality data (time of death) were obtained from the Federal Institute under Public Law "Statistics Austria". Pacemaker lead replacements were defined by implantation of at least one new lead (atrial or ventricular) with or without lead extraction within the clinical follow-up.

\section{Echocardiography}

Transthoracic echocardiography studies were performed before and after pacemaker implantation. All echocardiographic studies were performed by board-certified physicians, and high-end scanners (Vivid E9, Vivid7, GE Healthcare, Chicago/Illinois, USA) with 2.5-MHz transducers were used. The conducted 2-dimensional echo exams included parasternal, apical, and subcostal views with M-mode, 2-dimensional echocardiography, and conventional and color Doppler ultrasonography according to current recommendations [22, 23]. TR and mitral regurgitation were graded by visual estimation under consideration of an integrated approach including valve morphology, color flow jet, continuous wave signal of the jet, vena contracta width, and proximal isovelocity surface area (PISA) radius as recommended by the guidelines of the European Association of Echocardiography and the American Society of Echocardiography [24, 25], and were categorized in three groups: no or mild, moderate, and severe. Lead-associated progression of TR was defined by progressing of TR from no/mild to moderate or severe, or from moderate to severe comparing echocardiography studies before and after pacemaker implantation. RV was graded as dilated if the basal RV enddiastolic diameter was $42 \mathrm{~mm}$ or larger in 2D echocardiography (4-chamber view) [22, 23]. Further visual assessment of the RV was performed in parasternal long and short axis and subxiphoidal views. Right and left atria were graded as significantly dilated if the longitudinal diameter in the apical 4-chamber view exceeded $70.0 \mathrm{~mm}$. Left ventricular function (LVF) was categorized as normal, mildly [left ventricular ejection fraction (LVEF) 41-53\% in women and $41-51 \%$ in men], moderately (LVEF $30-40 \%$ ) or severely $(\mathrm{LVEF}<30 \%)$ impaired after visual estimation [22, 23].

Peak tricuspid regurgitation velocity (TR- $V_{\max }$ ) was assessed by continuous wave (CW) Doppler. Systolic pulmonary artery pressure (sPAP) was calculated as the sum of the tricuspid jet gradient (assessed by Doppler) and right atrial pressure. Right atrial pressure was estimated by visualizing the inferior vena cava and its response to respiration. Presence of significant $\mathrm{PH}$ was assumed if sPAP was $\geq 56 \mathrm{mmHg}$ or TR- $V_{\max }$ was $\geq 3.5 \mathrm{~m} / \mathrm{s}$.

\section{Statistical analysis}

Statistical analyses were performed with SPSS software (version 24.0; Macintosh; SPSS IBM). Continuous variables were tested for normal and non-normal distribution, and means \pm standard deviations were calculated. Groups with continuous variables were compared with the t-test (normal distribution) or with the Mann-Whitney $U$ test (non-normal distribution). Discrete variables were compared with the chi-squared test. Odds ratios for progression of TR were calculated with a logistic regression model. Predicting factors for survival (baseline echocardiographic and clinical parameters) were determined with COX regression with a follow-up of 10 years, and hazard ratio and $95 \%$ confidence intervals were reported. Parameters were included in the 
multifactorial regression models if the $P$ value was $<0.10$ in the univariate regression. A two-sided $P$ value $<0.05$ was considered statistically significant.

\section{Results}

\section{Baseline patient parameters}

Baseline characteristics of the 990 enrolled patients are presented in Table 1. In total, 747 patients had no RV dilatation, and 243 patients had RV dilatation at baseline.

Patients with RV dilatation had more atrial fibrillation and higher prevalence of indications for single-chamber pacemaker. Patients with RV dilatation had more CABG, but rates of coronary artery disease showed no difference. Heart failure was more common in patients with RV dilatation. Patients with RV dilatation exhibited a higher prevalence of chronic kidney disease.

Echocardiographic parameters are presented in Table 2. Patients with RV dilatation had more severe degrees of tricuspid regurgitation both pre- and post-intervention, and pre-interventional TR- $V_{\max }$ and SPAP were higher. Regarding left heart disease, the group with RV dilatation had

Table 1 Baseline patient characteristics of the study population

\begin{tabular}{lllc}
\hline & $\begin{array}{l}\text { No pre-existing } \\
\text { RV dilatation } \\
N=747\end{array}$ & $\begin{array}{l}\text { Pre-existing } \\
\text { RV dilatation } \\
N=243\end{array}$ & $P$ value \\
& & \\
\hline Age (years) & $70.4 \pm 12.5$ & $70.4 \pm 10.9$ & 0.95 \\
Female sex & $285(38.2 \%)$ & $92(37.9 \%)$ & 0.94 \\
BMI $\left(\mathrm{kg} / \mathrm{m}^{2}\right)$ & $26.5 \pm 4.7$ & $26.7 \pm 4.7$ & 0.67 \\
BSA $\left(\mathrm{m}^{2}\right)$ & $1.89 \pm 0.23$ & $1.89 \pm 0.23$ & 0.63 \\
Single-chamber pace- & $158(23.1 \%)$ & $82(40.8 \%)$ & $<\mathbf{0 . 0 0 1}$ \\
$\quad$ maker & & & \\
RV pacing threshold $(\mathrm{V})$ & $0.71 \pm 0.61$ & $0.75 \pm 0.63$ & 0.80 \\
Coronary artery disease & $420(56.2 \%)$ & $147(60.5 \%)$ & 0.24 \\
Heart failure & $375(50.2 \%)$ & $145(59.7 \%)$ & $\mathbf{0 . 0 1}$ \\
Diabetes & $191(25.6 \%)$ & $68(28.0 \%)$ & 0.46 \\
Myocarditis & $3(0.4 \%)$ & $1(0.4 \%)$ & 0.98 \\
Any atherosclerosis & $498(66.7 \%)$ & $161(66.3 \%)$ & 0.91 \\
Previous stroke or TIA & $94(12.6 \%)$ & $32(13.2 \%)$ & 0.81 \\
Previous CABG & $55(7.4 \%)$ & $38(15.6 \%)$ & $<\mathbf{0 . 0 0 1}$ \\
Atrial fibrillation & $385(51.5 \%)$ & $169(69.5 \%)$ & $<\mathbf{0 . 0 0 1}$ \\
Chronic kidney disease & $183(24.5 \%)$ & $95(39.1 \%)$ & $<\mathbf{0 . 0 0 1}$ \\
Endocarditis & $58(7.8 \%)$ & $22(9.1 \%)$ & 0.52 \\
\hline
\end{tabular}

Values are mean $\pm \mathrm{SD}, N(\%)$, or median (interquartile range), unless otherwise indicated. $P$ values $<0.05$ in bold

$B M I$ body mass index, $B S A$ body surface area, $C A B G$ coronary artery bypass grafting, $R V$ right ventricle, TIA transient ischemic attack, $V$ volt
Table 2 Echocardiographic patient profile

\begin{tabular}{|c|c|c|c|}
\hline & $\begin{array}{l}\text { No pre-existing } \\
\text { RV dilatation } \\
N=747\end{array}$ & $\begin{array}{l}\text { Pre-existing } \\
\text { RV dilatation } \\
N=243\end{array}$ & $P$ value \\
\hline \multicolumn{4}{|l|}{ Pre-pacemaker } \\
\hline No or mild TR & $579(77.5 \%)$ & $148(60.9 \%)$ & $<0.001$ \\
\hline Moderate TR & $131(17.5 \%)$ & $60(24.7 \%)$ & \\
\hline Severe TR & $37(5.0 \%)$ & $35(14.4 \%)$ & \\
\hline \multicolumn{4}{|l|}{ Post-pacemaker } \\
\hline No or mild TR & $476(63.7 \%)$ & $75(30.9 \%)$ & $<0.001$ \\
\hline Moderate TR & $216(28.9 \%)$ & $85(35.0 \%)$ & \\
\hline Severe TR & $55(7.4 \%)$ & $83(34.2 \%)$ & \\
\hline \multicolumn{4}{|l|}{ Pre-pacemaker } \\
\hline \multicolumn{4}{|l|}{ Left ventricular function } \\
\hline Normal & $431(57.8 \%)$ & $97(39.9 \%)$ & $<0.001$ \\
\hline Mild reduction & $121(16.2 \%)$ & $44(18.1 \%)$ & \\
\hline Moderate reduction & $100(13.4 \%)$ & $35(14.4 \%)$ & \\
\hline Severe reduction & $94(12.6 \%)$ & $67(27.6 \%)$ & \\
\hline \multicolumn{4}{|l|}{ Mitral regurgitation } \\
\hline No or mild & $343(53.3 \%)$ & $61(32.3 \%)$ & $<0.001$ \\
\hline Moderate & $254(39.4 \%)$ & $83(43.9 \%)$ & \\
\hline Severe & $47(7.3 \%)$ & $45(23.8 \%)$ & \\
\hline sPAP $(\mathrm{mmHg})$ & $44.7 \pm 14.2$ & $58.2 \pm 17.6$ & $<0.001$ \\
\hline LVEDD indexed $\left(\mathrm{mm} / \mathrm{m}^{2}\right)$ & $25.4 \pm 4.4$ & $27.2 \pm 5.4$ & $<0.001$ \\
\hline $\mathrm{LA}(\mathrm{mm})$ & $61 \pm 10$ & $70 \pm 11$ & $<0.001$ \\
\hline RVEDD (mm) & $33 \pm 4$ & $43 \pm 6$ & $<0.001$ \\
\hline \multicolumn{4}{|l|}{ Right ventricular function } \\
\hline Normal & $598(85.2 \%)$ & $86(39.4 \%)$ & $<0.001$ \\
\hline Mild reduction & $76(10.8 \%)$ & $64(29.4 \%)$ & \\
\hline Moderate reduction & $21(3.0 \%)$ & $48(22.0 \%)$ & \\
\hline Severe reduction & $7(1.0 \%)$ & $20(9.2 \%)$ & \\
\hline $\mathrm{RA}(\mathrm{mm})$ & $59 \pm 10$ & $72 \pm 45$ & $<0.001$ \\
\hline $\mathrm{TR}-V_{\max }(\mathrm{m} / \mathrm{s})$ & $2.93 \pm 0.64$ & $3.09 \pm 0.65$ & 0.004 \\
\hline
\end{tabular}

Values are mean $\pm \mathrm{SD}, N(\%)$, or median (interquartile range), unless otherwise indicated. $P$ values $<0.05$ in bold

$L A$ left atrium, $L V E D D$ left ventricular end-diastolic diameter, $R A$ right atrium, $R V$ right ventricle, $R V E D D$ right ventricular end-diastolic diameter, $S P A P$ systolic pulmonary artery pressure, $T R$ tricuspid regurgitation, $T R-V_{\max }$ peak tricuspid regurgitation velocity

worse LVF, higher rates of mitral regurgitation, and larger dimensions of the left ventricle and the left atrium.

\section{Progression of tricuspid regurgitation}

In total, 303 out of 990 patients (30.6\%) had progression of TR after pacemaker lead implantation, as shown in Fig. 1 and in Supplemental Fig. 1. Representative echocardiography studies evaluating TR before and after pacemaker implantation are displayed in Fig. 2. Pacemaker leadassociated progression of TR occurred in 112 out of 243 patients with RV dilatation (46.1\%) compared to 191 out of 
Fig. 1 Tricuspid regurgitation before and after implantation of pacemaker. Sankey chart of grade of pre-existing TR with visualized flow to post-interventional TR after pacemaker implantation. Green: no/mild TR, orange: moderate TR, red: severe TR. Left: patients without RV dilatation. Right: patients with RV dilatation. Grades of TR are displayed in \%. $P M$ pacemaker, TR tricuspid regurgitation

Fig. 2 Echocardiography images evaluating tricuspid regurgitation before and after pacemaker implantation. Representative images from transthoracic echocardiography (4-chamber view) evaluating tricuspid regurgitation before and after pacemaker implantation in a patient without right ventricular dilatation (left column) and in a patient with right ventricular dilatation (right column)

\section{No pre-existing RV Dilatation $N=747$}

TR before PM TR after PM

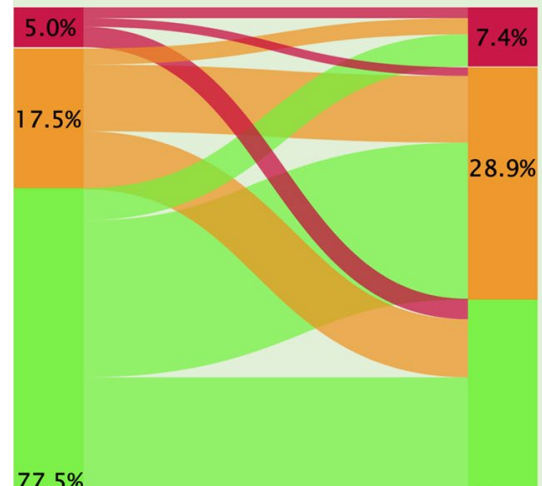

$63.7 \%$

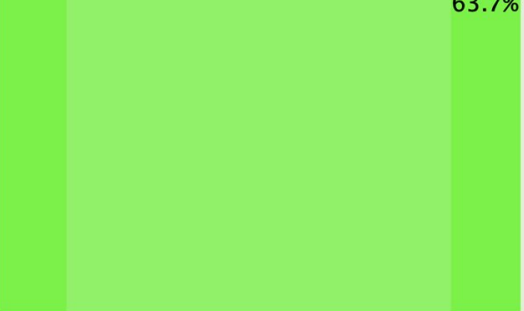

\section{Pre-existing RV Dilatation}

$N=243$

TR before PM

TR after PM

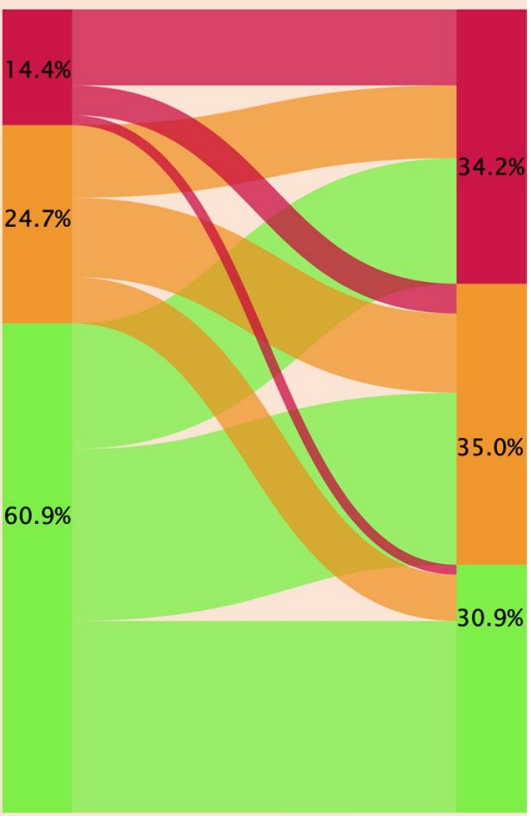

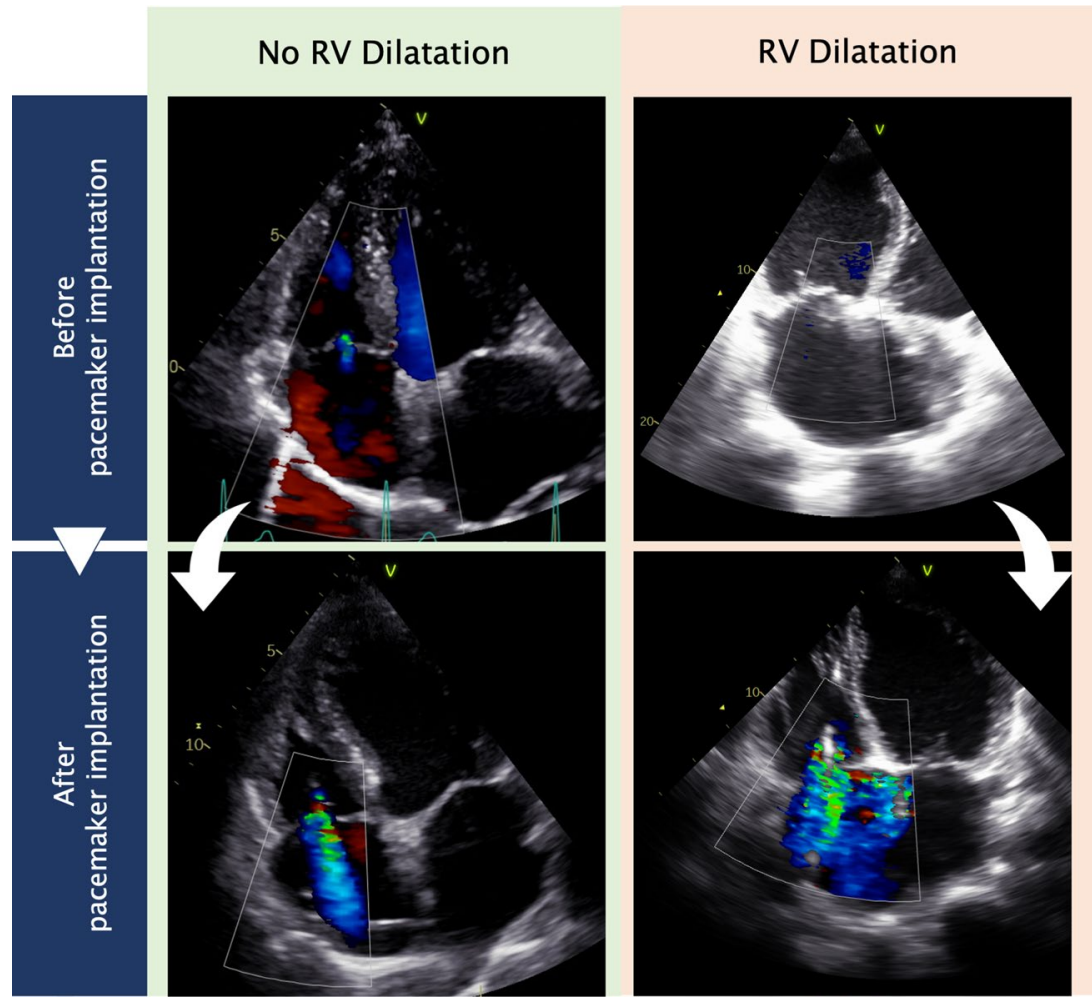

747 patients without RV dilatation $(25.6 \% ; P<0.001)$. The odds ratio (univariate) for progression of TR was increased if RV dilatation was prevalent (OR 2.49; 95\% CI 1.84-3.36; $P<0.001$, compared to normal RV dimensions).
Using an ordinal regression model, the probability to suffer from severe TR after pacemaker implantation in patients with no or mild pre-existing TR and without RV dilatation was $6.3 \%$ (95\% CI 4.7-8.3\%), compared to $21.5 \%$ (95\% CI 
21.4-21.6\%) in patients with RV dilatation. The probability to suffer from severe TR after pacemaker implantation in patients with pre-existing moderate TR and without RV dilatation was $14.8 \%$ (95\% CI 11.0-19.7\%), compared to $41.6 \%$ (95\% CI 40.3-42.8\%) in patients with RV dilatation.

Improvement of TR of at least one grade after pacemaker implantation occurred in $10.7 \%(N=26)$ of patients with RV dilatation and in $10.8 \%(N=81)$ of patients without $\mathrm{RV}$ dilatation $(P=0.95)$.

\section{Risk factors for progression of TR}

Risk factors for progression of TR are shown in Table 3. After adjustment for patient baseline characteristics and echocardiographic parameters, the following characteristics were independently associated with progression of TR: RV dilatation (OR 2.04; 95\% CI 1.27-3.29; $P=0.003$ ), moderate pre-existing TR (OR 4.30; 95\% CI 2.51-7.38; $P<0.001)$, female sex (OR 1.68; 95\% CI 1.16-2.43; $P=0.006$ ), single RV lead (OR 1.67; 95\% CI 1.09-2.56; $P=0.018$ ), moderate or severe mitral regurgitation (OR 2.08; 95\% CI 1.42-3.05; $P<0.001$ ), and an enlarged left atrium of $\geq 70 \mathrm{~mm}$ (OR $1.98 ; 95 \%$ CI $1.07-3.67 ; P=0.03$ ).

\section{Mortality}

During the follow-up, 5569 person-years were analyzed, and the median follow-up time was 5.6 years (IQR 2.8-8.9 years). Completed 10-year follow-up was available for 562 of 990 patients, with a mortality rate of $62.2 \%$ of these 562 patients. Figure 3 indicates survival shown by Kaplan-Meier plot.

In patients without RV dilatation, lead-associated progression of TR had a higher mortality rate compared to nonprogressors (HR 1.35; 95\% CI 1.03-1.76; $P=0.028$ ). The 10 -year survival rate was $68.5 \%$ vs. $59.2 \%$ (non-progressors vs. progressors without RV dilatation). Patients with RV dilatation had no statistically significant differences in survival if lead-associated progression of TR was present (HR 1.33; 95\% CI 0.93-1.89; $P=0.12$ ). The 10 -year survival rate was $54.2 \%$ vs. $45.5 \%$ (non-progressors vs. progressors with RV dilatation).

Table 4 shows the results of univariate and multivariate COX regression. After adjustment for relevant comorbidities and echocardiographic parameters, independent factors for decreased survival were pacemaker lead-associated TR (HR 1.38 ; 95\% CI 1.04-1.84; $P=0.028$ ), moderate/severe mitral regurgitation (HR 1.34; 95\% CI 1.02-1.77; $P=0.034$ ), heart
Table 3 Predictors for progression of tricuspid regurgitation

\begin{tabular}{|c|c|c|c|c|}
\hline & \multicolumn{2}{|l|}{ Univariate } & \multicolumn{2}{|l|}{ Multivariate } \\
\hline & OR $(95 \% \mathrm{CI})$ & $P$ value & OR $(95 \% \mathrm{CI})$ & $P$ value \\
\hline RV Dilatation & $2.49(1.84-3.36)$ & $<0.001$ & $2.04(1.27-3.29)$ & 0.003 \\
\hline RVF Reduction & $1.66(1.22-2.27)$ & 0.001 & $0.97(0.60-1.56)$ & 0.890 \\
\hline Pre-existing TR (moderate) & $2.08(1.41-3.06)$ & $<0.001$ & $4.30(2.51-7.38)$ & $<0.001$ \\
\hline Age $\geq 80$ & $1.11(0.79-1.54)$ & 0.551 & & \\
\hline Female sex & $1.39(1.06-1.83)$ & 0.018 & $1.68(1.16-2.43)$ & 0.006 \\
\hline Single RV lead & $1.62(1.18-2.21)$ & 0.003 & $1.67(1.09-2.56)$ & 0.018 \\
\hline Coronary artery disease & $1.36(1.03-1.79)$ & 0.031 & $0.98(0.49-1.94)$ & 0.950 \\
\hline Heart failure & $1.68(1.28-2.21)$ & $<0.001$ & $1.39(0.88-2.19)$ & 0.159 \\
\hline Diabetes & $0.99(0.73-1.35)$ & 0.966 & & \\
\hline Any atherosclerosis & $1.28(0.96-1.71)$ & 0.099 & $1.13(0.56-2.28)$ & 0.726 \\
\hline Atrial fibrillation & $1.58(1.20-2.09)$ & 0.001 & $1.15(0.78-1.69)$ & 0.474 \\
\hline Chronic kidney disease & $1.35(1.003-1.81)$ & 0.048 & $1.04(0.71-1.54)$ & 0.831 \\
\hline Endocarditis & $1.48(0.93-2.38)$ & 0.101 & & \\
\hline $\begin{array}{l}\text { Mitral regurgitation (moderate } \\
\text { or severe) }\end{array}$ & $2.22(1.63-3.02)$ & $<0.001$ & $2.08(1.42-3.05)$ & $<0.001$ \\
\hline $\mathrm{sPAP} \geq 56 \mathrm{mmHg}$ & $2.16(1.47-3.15)$ & $<0.001$ & $1.18(0.69-2.05)$ & 0.546 \\
\hline LVEDD $\geq 25 \mathrm{~mm} / \mathrm{m}^{2}$ & $1.33(1.01-1.75)$ & 0.04 & $1.09(0.75-1.57)$ & 0.663 \\
\hline $\mathrm{LA} \geq 70 \mathrm{~mm}$ & $2.30(1.69-3.13)$ & $<0.001$ & $1.98(1.07-3.67)$ & $\mathbf{0 . 0 3 0}$ \\
\hline $\mathrm{RA} \geq 70 \mathrm{~mm}$ & $2.03(1.47-2.82)$ & $<0.001$ & $0.75(0.38-1.46)$ & 0.396 \\
\hline
\end{tabular}

List of parameters included in the uni- and multivariate regression. All echocardiographic parameters were obtained from the echocardiographic study before pacemaker implantation. Parameters were included in the multivariate regression model with a $P$ value $<0.10$ in the univariate regression

$C I$ confidence interval, $L A$ left atrium, $L V E D D$ left ventricular end-diastolic diameter, $O R$ odds ratio, $R A$ right atrium, $R V$ right ventricle, $R V F$ right ventricular function, $s P A P$ systolic pulmonary artery pressure, $T R$ tricuspid regurgitation 
Fig. 3 Survival of patients with/ without right ventricular dilatation and with/without pacemaker lead-associated progression of tricuspid regurgitation. Kaplan-Meier plot of included patients with a 10-year followup. No. of events and patients at risk (No. at risk) are given in total numbers at year $0,2,4,6$, 8 , and $10 . R V$ right ventricle, $T R$ tricuspid regurgitation

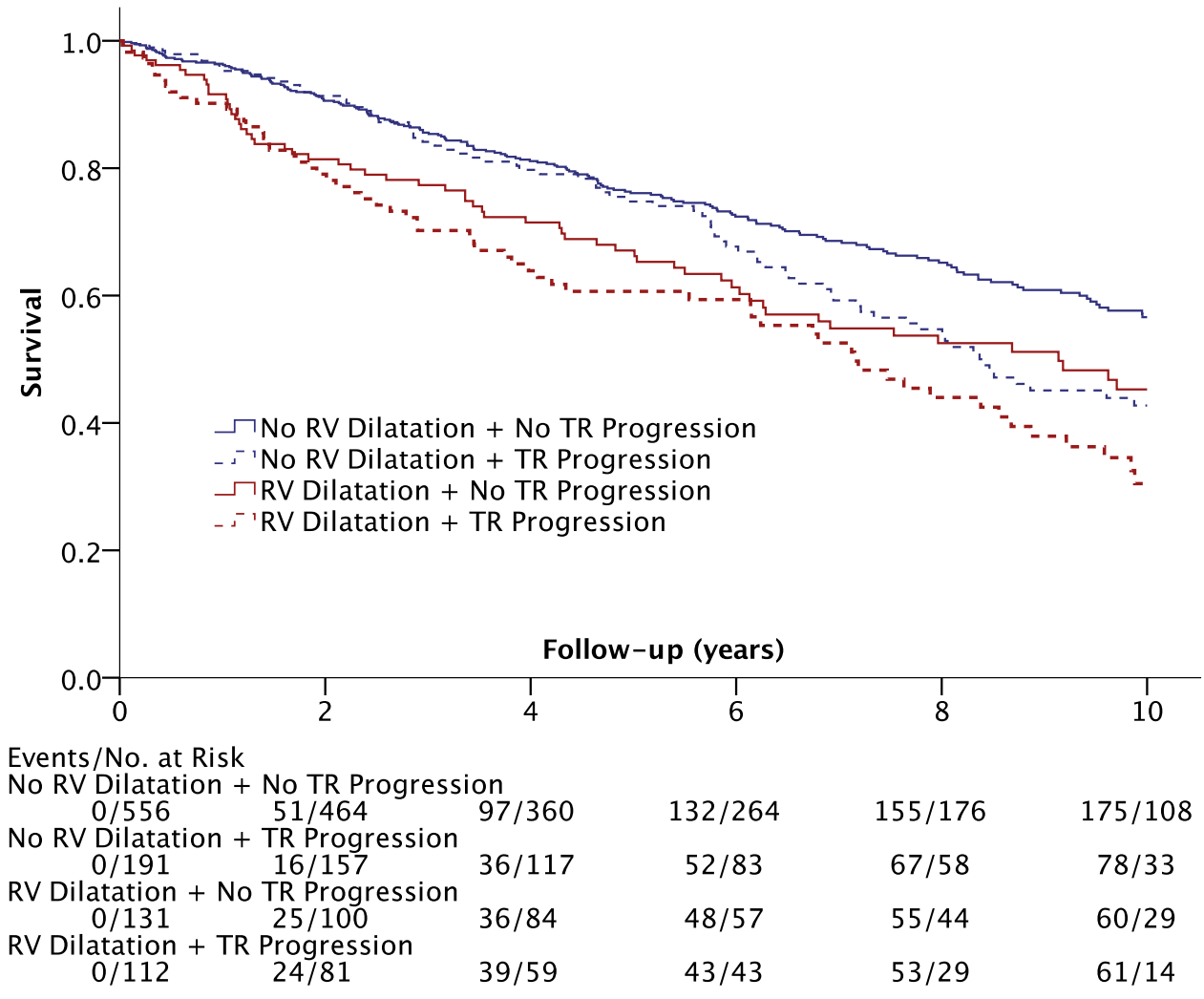

failure (HR 1.75; 95\% CI 1.31-2.33; $P<0.001$ ), chronic kidney disease (HR 1.62; 95\% CI 1.25-2.11; $P=0.001$ ), and age $\geq 80$ years (HR 2.84; 95\% CI 2.17-3.71; $P<0.001$ ).

\section{Lead replacement rate}

Replacement of at least one pacemaker lead was necessary in 94 out of 990 patients (9.5\%). Patients with RV dilatation had a higher risk for lead replacement $(12.8 \%$ of patients with RV dilatation vs. $8.4 \%$ of patients without RV dilatation, OR $1.59 ; 95 \%$ CI $1.01-2.51 ; P=0.047)$. Neither the grade of TR before or after PM implantation nor the progression of TR was associated with higher risks for lead replacement.

\section{Discussion}

This is the first study investigating the link between RV dimensions and the risk of pacemaker lead-associated progression of TR. The primary findings are: (1) Patients with prior RV dilatation had an increased probability of progression of TR. (2) Beside RV dimensions, independent predictors for pacemaker lead-associated TR were pre-existing TR (at least moderate), female sex, single RV lead, and moderate/severe mitral regurgitation. (3) Independent survival predictors included lead-associated TR, mitral regurgitation, heart failure, chronic kidney disease, and age $\geq 80$ years.

Previous studies have reported a high incidence of TR progression after CIED implantation. In our study, 30.6\% of patients exhibited progression of TR after pacemaker implantation, which is comparable to $38 \%$ reported by Höke et al. [16], but significantly higher than the $21.2 \%$ found by Kim et al. [26], 18.3\% found by Klutstein et al. [27], or $13 \%$ found by Seo et al. [19].

The main reason for the discrepancies between these studies lies in differing definitions of significant TR and of progression of TR. Our study included all grades of TR, had liberal definitions of progression of TR, and is most comparable to the design and definitions of Höke et al.: All grades of TR were included, and the echocardiography were performed from 1 to 1.5 years after CIED implantation, which is comparable to our study [16]. Compared to Höke et al., our analysis included more patients with longer follow-up and excluded patients with ICD or CRT, resulting in a more homogenous patient collective with focus on the properties of the right ventricle.

Seo et al. reported a rate of TR progression of only $13 \%$, which is the lowest value reported in all comparable studies [19]. Importantly, they excluded all patients with moderate or severe TR before CIED implantation, and defined worsened TR by progression to moderate or severe TR after 
Table 4 COX regression identifying predictors for mortality

\begin{tabular}{|c|c|c|c|c|}
\hline & \multicolumn{2}{|l|}{ Univariate } & \multicolumn{2}{|l|}{ Multivariate } \\
\hline & HR $(95 \%$ CI $)$ & $P$ value & $\operatorname{HR}(95 \% \mathrm{CI})$ & $P$ value \\
\hline RV Dilatation & $1.58(1.27-1.96)$ & $<0.001$ & $0.95(0.67-1.33)$ & 0.747 \\
\hline RVF Reduction & $1.58(1.25-2.00)$ & $<0.001$ & $1.14(0.83-1.57)$ & 0.413 \\
\hline Pre-existing TR (moderate) & $1.34(1.07-1.67)$ & 0.01 & $1.30(0.94-1.81)$ & 0.116 \\
\hline Lead-associated TR progression & $1.44(1.17-1.78)$ & 0.001 & $1.38(1.04-1.84)$ & 0.028 \\
\hline Age $\geq 80$ & $2.78(2.23-3.47)$ & $<0.001$ & $2.84(2.17-3.71)$ & $<0.001$ \\
\hline Female sex & $0.87(0.71-1.08)$ & 0.202 & & \\
\hline Single RV lead & $1.26(0.99-1.60)$ & 0.061 & $1.27(0.96-1.69)$ & 0.093 \\
\hline Coronary artery disease & $1.10(0.90-1.36)$ & 0.35 & & \\
\hline Heart failure & $1.38(1.12-1.69)$ & 0.002 & $1.75(1.31-2.33)$ & $<0.001$ \\
\hline Diabetes & $1.13(0.90-1.41)$ & 0.289 & & \\
\hline Any atherosclerosis & $0.99(0.80-1.23)$ & 0.944 & & \\
\hline Atrial fibrillation & $0.92(0.75-1.12)$ & 0.392 & & \\
\hline Chronic kidney disease & $1.55(1.25-1.92)$ & $<0.001$ & $1.62(1.25-2.11)$ & $<0.001$ \\
\hline Endocarditis & $0.67(0.44-1.02)$ & 0.061 & $1.10(0.61-1.99)$ & 0.749 \\
\hline Mitral regurgitation (moderate or severe) & $1.90(1.51-2.37)$ & $<0.001$ & $1.34(1.02-1.77)$ & $\mathbf{0 . 0 3 4}$ \\
\hline $\mathrm{sPAP} \geq 56 \mathrm{mmHg}$ & $0.95(0.68-1.32)$ & 0.736 & & \\
\hline LVEDD $\geq 25 \mathrm{~mm} / \mathrm{m} 2$ & $1.59(1.29-1.96)$ & $<0.001$ & $1.29(0.997-1.67)$ & 0.053 \\
\hline $\mathrm{LA} \geq 70 \mathrm{~mm}$ & $1.21(0.96-1.52)$ & 0.108 & & \\
\hline $\mathrm{RA} \geq 70 \mathrm{~mm}$ & $1.23(0.97-1.56)$ & 0.09 & $0.84(0.60-1.18)$ & 0.308 \\
\hline $\mathrm{TR}-V_{\max } \geq 3.5 \mathrm{~m} / \mathrm{s}$ & $1.55(1.18-2.05)$ & 0.002 & $0.99(0.65-1.49)$ & 0.945 \\
\hline
\end{tabular}

List of parameters included in the uni- and multivariate COX regression. All echocardiographic parameters were obtained from the echocardiographic study before pacemaker implantation. Parameters were included in the multivariate $\mathrm{COX}$ regression with a $P$ value $<0.10$ in the univariate $\mathrm{COX}$ regression

$C I$ confidence interval, $H R$ hazard ratio, $L A$ left atrium, $L V E D D$ left ventricular end-diastolic diameter, $R A$ right atrium, $R V$ right ventricle, $R V F$ right ventricular function, $s P A P$ systolic pulmonary artery pressure, $T R$ tricuspid regurgitation
CIED implantation. Our study included the whole spectrum of TR, which explains the discrepancy.

Our study cohort exclusively contains patients with pacemakers, as patients with CRTs or ICDs were excluded. Inclusion of CRT would skew the results because of modified post-interventional rates of mitral regurgitation due to enhanced LVF. Furthermore, biventricular pacing may modify the risk of secondary CIED-related TR. The increased thickness and stiffness of ICD leads have been reported to cause higher rates of lead-associated TR, which is the reason for exclusion from our cohort [26]. Additionally, patients with CRTs or ICDs generally suffer from lower LVF and increased mortality compared to pacemaker patients [28].

Atrial fibrillation was found to represent a risk factor for CIED-associated progression of TR [17, 29]. In our univariate regression for progression of TR, atrial fibrillation and single ventricular pacing lead was found to be significantly associated with progression of TR. In the multivariate analysis, only single ventricular pacing lead remained as a risk factor. This could be still due to atrial fibrillation, as atrial fibrillation is the major indication for implantation of a pacemaker with a single ventricular lead. Atrial fibrillation is a common driver for TR due to its dilatating effect on the right atrium, triggering coaptation defects of the tricuspid valve [30].

\section{Risk factors for progression of TR}

Delling et al. reported no significant progression of TR after CIED implantation in 169 patients, but described independent risk factors for TR in patients with CIED, which included age, body mass index, heart rate, right ventricular dilatation, $\mathrm{sPAP} \geq 37 \mathrm{mmHg}$, history of mitral valve repair or replacement, and severe mitral regurgitation. Although the number of patients with echocardiography both before and after CIED implantation was low $(N=169)$, the study included 1245 patients with echocardiography only after CIED implantation. Analysis of that cohort revealed similar risk factors for TR with CIED, but lacked a direct comparison of TR before and after implantation. Additionally, analysis of mortality did not include right ventricular dilatation. In this context, our study provides substantial new insights regarding CIEDs, $\mathrm{TR}$, and right ventricular properties, especially concerning $\mathrm{RV}$ dilatation. 


\section{Survival}

Association of CIED-induced progression of TR and mortality is well-known, and worsening of TR has been identified as an independent risk factor for mortality. Our observed hazard ratio of 1.38 for pacemaker lead-associated TR is comparable to the hazard ratio of 1.65 reported by Höke et al., and of 1.40 by Delling et al. The study by Seo et al. described a higher hazard ratio of 2.8 for TR progression after CIED implantation, but this is likely due to their stringent definition of TR progression.

\section{Improved TR after pacemaker implantation}

Amelioration of TR after pacemaker implantation is occasionally seen. Whether TR improves after CIED mplantation depends on the hemodynamic severity of bradycardic disorders prior to CIED implantation [14, 27, 31]. After pacemaker implantation, the cardiac output may increase, the right ventricular pressure decreases, leading to improved TR in some patients.

\section{Clinical consequences}

Better understanding of independent risk factors for pacemaker lead-associated TR and the associated increase in mortality might enable better risk stratification and therefore a more personalized treatment approach in patients with indication for pacemakers.

Possible options for patients with expected pacemaker lead-associated TR could include leadless pacing, HIS-bundle pacing or coronary sinus pacing. In a recent small-scale study, leadless pacing caused worsening of TR in $43 \%$ of 53 patients after a follow-up of 12 months [32]. This counterintuitive finding defies expectations but has yet to be replicated in larger trials. A possible mechanism might be functional TR caused by dyssynchrony.

If pacemaker lead-associated TR occurs, TTVI is an attractive option, as Taramasso et al. demonstrated feasibility and good clinical outcomes of TTVI in patients with TR and CIED [21]. However, some patients with primary severe CIED-induced TR may require surgery [33].

\section{Strengths}

The main strengths of this study are the large sample size and the extended longitudinal follow-up with thorough survival analysis, and the definition of predictive factors for pacemaker lead-associated TR, facilitating personalized decision for TTVI.

\section{Limitations}

The major limitation of this study is its retrospective nature. Residual confounding and selection bias might be an issue, highlighting the urgency for prospective trials. Patients without post-interventional echocardiography were not included in this trial. Some clinical characteristics were identified by ICD-10 codes, which are reliant on accurate recording by treating physicians. Although RV dilatation and TR are known drivers for further progression of TR independently of transtricuspid pacing leads, pacemaker leads represent a major independent risk factor for TR progression in a matched RV dilatation-cohort comparing patients with vs. without pacemakers. The only study from the literature which compares a large cohort of patients with and without pacemakers is cross-sectional hence can only describe a statistical instead of a functional association between transvenous pacemaker leads and both tricuspid regurgitation and mortality [15]. Patients with RV dilatation were in overall sicker than patients without RV dilatation. Our data on RV dilation and TR lack a control group of patients without pacemaker, which could be an acceptable method to adjust for contributing factors for progression of TR in patients (i.e., RV dilatation or pre-existing TR). Unfortunately, this study lack data from 3D-TTE, leading to the fact that the exact mechanism of progression of TR remained unknown and true severity of tricuspid regurgitation after pacemaker implantation could be underestimated. Reliable clinical 3D-TTE was not available in the majority of the included patients, as inclusion of patients started in the year 2000. Detailed echocardiographic data such as left ventricular ejection fraction (in \%) or detailed measurements of TR (vena contracta, proximal isovelocity surface area) were not available, and interobserver variability has to be considered as a potential bias in studies based on echo data. Although data for lead replacements were analyzed, the reasons for the lead replacement were not available.

\section{Conclusion}

Patients with prior RV dilatation suffered from higher rates of TR progression after pacemaker implantation, and patients with pacemaker lead-associated progression of TR exhibited impaired survival. Patients at risk for pacemaker lead-associated progression of TR could be treated with leadless pacemakers, and TTVI has to be further evaluated as a treatment strategy for patients with pacemaker lead-associated TR. 
Funding Open access funding provided by Medical University of Vienna. This study was funded by the European Union's Horizon 2020 Future and Emerging Technologies Programme (Grant number 732170).

\section{Compliance with ethical standards}

Conflict of interest Martin Andreas has served as a Proctor for Edwards and Abbott, served as an Advisor for Medtronic and reported receiving Institutional Grants from LSI, Abbott, Edwards, and Medtronic during the conduct of the study. All other authors have reported that they have no relationships relevant to the contents of this paper to disclose.

Open Access This article is licensed under a Creative Commons Attribution 4.0 International License, which permits use, sharing, adaptation, distribution and reproduction in any medium or format, as long as you give appropriate credit to the original author(s) and the source, provide a link to the Creative Commons licence, and indicate if changes were made. The images or other third party material in this article are included in the article's Creative Commons licence, unless indicated otherwise in a credit line to the material. If material is not included in the article's Creative Commons licence and your intended use is not permitted by statutory regulation or exceeds the permitted use, you will need to obtain permission directly from the copyright holder. To view a copy of this licence, visit http://creativecommons.org/licenses/by/4.0/.

\section{References}

1. Topilsky Y, Maltais S, Medina Inojosa J et al (2019) Burden of tricuspid regurgitation in patients diagnosed in the community setting. JACC Cardiovasc Imaging 12:433-442. https://doi. org/10.1016/j.jcmg.2018.06.014

2. Rodés-Cabau J, Taramasso M, O'Gara PT (2016) Diagnosis and treatment of tricuspid valve disease: current and future perspectives. Lancet 388:2431-2442. https://doi.org/10.1016/S0140 -6736(16)00740-6

3. Nath J, Foster E, Heidenreich PA (2004) Impact of tricuspid regurgitation on long-term survival. J Am Coll Cardiol 43:405-409. https://doi.org/10.1016/j.jacc.2003.09.036

4. Nishimura RA, Otto CM, Bonow RO et al (2017) 2017 AHA/ACC focused update of the 2014 AHA/ACC guideline for the management of patients with valvular heart disease. J Am Coll Cardiol 70:252-289. https://doi.org/10.1016/j.jacc.2017.03.011

5. Baumgartner H, Falk V, Bax JJ et al (2017) 2017 ESC/EACTS guidelines for the management of valvular heart disease. Eur Heart J 38:2739-2791. https://doi.org/10.1093/eurheartj/ehx391

6. Asmarats L, Puri R, Latib A et al (2018) Transcatheter tricuspid valve interventions. J Am Coll Cardiol 71:2935-2956. https://doi. org/10.1016/j.jacc.2018.04.031

7. Taramasso M, Pozzoli A, Guidotti A et al (2016) Percutaneous tricuspid valve therapies: the new frontier. Eur Heart J. https://doi. org/10.1093/eurheartj/ehv766

8. Taramasso M, Alessandrini H, Latib A et al (2019) Outcomes after current transcatheter tricuspid valve intervention. JACC Cardiovasc Interv 12:155-165. https://doi.org/10.1016/j. jcin.2018.10.022

9. Nickenig G, Weber M, Schueler R et al (2019) 6-Month outcomes of tricuspid valve reconstruction for patients with severe tricuspid regurgitation. J Am Coll Cardiol 73:1905-1915. https://doi. org/10.1016/j.jacc.2019.01.062
10. Orban M, Rommel K-P, Ho EC et al (2020) Transcatheter edgeto-edge tricuspid repair for severe tricuspid regurgitation reduces hospitalizations for heart failure. JACC Heart Fail 8:265-276. https://doi.org/10.1016/j.jchf.2019.12.006

11. Addetia K, Harb SC, Hahn RT et al (2019) Cardiac implantable electronic device lead-induced tricuspid regurgitation. JACC Cardiovasc Imaging 12:622-636. https://doi.org/10.1016/j. jcmg.2018.09.028

12. Chang JD, Manning WJ, Ebrille E, Zimetbaum PJ (2017) Tricuspid valve dysfunction following pacemaker or cardioverterdefibrillator implantation. J Am Coll Cardiol 69:2331-2341. https ://doi.org/10.1016/j.jacc.2017.02.055

13. Arabi P, Özer N, Ateş AH et al (2015) Effects of pacemaker and implantable cardioverter defibrillator electrodes on tricuspid regurgitation and right sided heart functions. Cardiol J 22:637644. https://doi.org/10.5603/CJ.a2015.0060

14. Al-Bawardy R, Krishnaswamy A, Rajeswaran J et al (2015) Tricuspid regurgitation and implantable devices: TR AND IMPLANTABLE DEVICES. Pacing Clin Electrophysiol 38:259266. https://doi.org/10.1111/pace.12530

15. Delling FN, Hassan ZK, Piatkowski G et al (2016) Tricuspid regurgitation and mortality in patients with transvenous permanent pacemaker leads. Am J Cardiol 117:988-992. https://doi. org/10.1016/j.amjcard.2015.12.038

16. Höke U, Auger D, Thijssen J et al (2014) Significant lead-induced tricuspid regurgitation is associated with poor prognosis at longterm follow-up. Heart 100:960-968. https://doi.org/10.1136/heart jnl-2013-304673

17. Seo J, Kim D-Y, Cho I et al (2020) Prevalence, predictors, and prognosis of tricuspid regurgitation following permanent pacemaker implantation. PLoS ONE 15:e0235230. https://doi. org/10.1371/journal.pone.0235230

18. Papageorgiou N, Falconer D, Wyeth N et al (2020) Effect of tricuspid regurgitation and right ventricular dysfunction on long-term mortality in patients undergoing cardiac devices implantation: > 10-year follow-up study. Int J Cardiol. https://doi.org/10.1016/j. ijcard.2020.05.062

19. Seo Y, Nakajima H, Ishizu T et al (2020) Comparison of outcomes in patients with heart failure with versus without lead-induced tricuspid regurgitation after cardiac implantable electronic devices implantations. Am J Cardiol 130:85-93. https://doi.org/10.1016/j. amjcard.2020.05.039

20. Nazmul MN, Cha Y-M, Lin G et al (2013) Percutaneous pacemaker or implantable cardioverter-defibrillator lead removal in an attempt to improve symptomatic tricuspid regurgitation. EP Eur 15:409-413. https://doi.org/10.1093/europace/eus342

21. Taramasso M, Gavazzoni M, Pozzoli A et al (2020) Outcomes of TTVI in patients with pacemaker or defibrillator leads. JACC Cardiovasc Interv 13:554-564. https://doi.org/10.1016/j. jcin.2019.10.058

22. Lang RM, Badano LP, Mor-Avi V et al (2015) Recommendations for cardiac chamber quantification by echocardiography in adults: an update from the American Society of Echocardiography and the European Association of Cardiovascular Imaging. Eur Heart J Cardiovasc Imaging 16:233-271. https://doi.org/10.1093/ehjci/ jev014

23. Rudski LG, Lai WW, Afilalo J et al (2010) Guidelines for the echocardiographic assessment of the right heart in adults: a report from the American Society of Echocardiography. J Am Soc Echocardiogr 23:685-713. https://doi.org/10.1016/j.echo.2010.05.010

24. Lancellotti P, Tribouilloy C, Hagendorff A et al (2013) Recommendations for the echocardiographic assessment of native valvular regurgitation: an executive summary from the European Association of Cardiovascular Imaging. Eur Heart J Cardiovasc Imaging 14:611-644. https://doi.org/10.1093/ehjci/jet105 
25. Schneider M, Binder T (2018) Echocardiographic evaluation of the right heart. Wien Klin Wochenschr 130:413-420. https://doi. org/10.1007/s00508-018-1330-3

26. Kim JB, Spevack DM, Tunick PA et al (2008) The effect of transvenous pacemaker and implantable cardioverter defibrillator lead placement on tricuspid valve function: an observational study. J Am Soc Echocardiogr 21:284-287. https://doi.org/10.1016/j. echo.2007.05.022

27. Klutstein M, Balkin J, Butnaru A et al (2009) Tricuspid incompetence following permanent pacemaker implantation. Pacing Clin Electrophysiol 32:S135-S137. https://doi.org/10.111 $1 / \mathrm{j} .1540-8159.2008 .02269 . x$

28. Dickstein K, Normand C, Auricchio A et al (2018) CRT Survey II: a European Society of Cardiology survey of cardiac resynchronisation therapy in 11088 patients-who is doing what to whom and how? CRT Survey II. Eur J Heart Fail 20:1039-1051. https://doi. org/10.1002/ejhf.1142

29. Cho MS, Kim J, Lee J-B et al (2019) Incidence and predictors of moderate to severe tricuspid regurgitation after dual-chamber pacemaker implantation. Pacing Clin Electrophysiol 42:85-92. https://doi.org/10.1111/pace.13543
30. Najib MQ, Vinales KL, Vittala SS et al (2012) Predictors for the development of severe tricuspid regurgitation with anatomically normal valve in patients with atrial fibrillation: severe functional TR in AF. Echocardiography 29:140-146. https://doi.org/10.111 1/j.1540-8175.2011.01565.x

31. Lee RC, Friedman SE, Kono AT et al (2015) Tricuspid regurgitation following implantation of endocardial leads: incidence and predictors: tricuspid regurgitation following endocardial leads. Pacing Clin Electrophysiol 38:1267-1274. https://doi. org/10.1111/pace.12701

32. Beurskens NEG, Tjong FVY, de Bruin-Bon RHA et al (2019) Impact of leadless pacemaker therapy on cardiac and atrioventricular valve function through 12 months of follow-up. Circ Arrhythm Electrophysiol. https://doi.org/10.1161/CIRCE P.118.007124

33. Andreas M, Gremmel F, Habertheuer A et al (2015) Case report: pacemaker lead perforation of a papillary muscle inducing severe tricuspid regurgitation. J Cardiothorac Surg. https://doi. org/10.1186/s13019-015-0244-7 